\title{
Integration of perioperative reduction treatment with lymphaticovenular anastomosis for the management of lower extremity lymphedema
}

\author{
Fumio Onishi ${ }^{1}$, Wataru Tsugu ${ }^{1}$, Nanae Okuda ${ }^{2}$, Toshiharu Minabe ${ }^{1}$ \\ 'Department of Plastic Surgery, Saitama Medical Center, Saitama Medical University, Kawagoe, Saitama 3508550, Japan. \\ ${ }^{2}$ Nursing Department, Tochigi Cancer Center, Utsunomiya, Tochigi 3200834, Japan. \\ Correspondence to: Assoc. Prof. Fumio Onishi, Department of Plastic Surgery, Saitama Medical Center, Saitama Medical \\ University, 1981 Kamoda, Kawagoe, Saitama 350-8550, Japan. E-mail: foonishi@saitama-med.ac.jp

\begin{abstract}
How to cite this article: Onishi F, Tsugu W, Okuda N, Minabe T. Integration of perioperative reduction treatment with lymphaticovenular anastomosis for the management of lower extremity lymphedema. Plast Aesthet Res 2021;8:50.
\end{abstract} \\ https://dx.doi.org/10.20517/2347-9264.2021.75
}

Received: 24 Jun 2021 First Decision: 14 Jul 2021 Revised: 23 Jul 2021 Accepted: 3 Aug 2021 Published: 11 Sep 2021

Academic Editor: Matthew L lorio Copy Editor: Xi-Jun Chen Production Editor: Xi-Jun Chen

\begin{abstract}
Aim: This study aimed to clarify the efficacy of the integration of lymphaticovenular anastomosis (LVA) and perioperative reduction treatment in the exploration of optimal combination of surgery and conservative therapy for lymphedema.

Methods: We conducted a retrospective chart review of 134 consecutive patients with lower extremity lymphedema who were treated with LVA. A total of 116 patients were included, and they were divided into two groups: patients who underwent perioperative reduction treatment (PORT) following LVA surgery (PORT group, 51 patients) and patients who underwent no additional perioperative intervention after LVA surgery (control group, 65 patients). PORT included compression therapy and remedial exercise. A total of 41 matched pairs were extracted after propensity score matching analysis. The edema reduction effect was compared between the two groups.
\end{abstract}

Results: Patients who underwent PORT had a significantly higher edema reduction than those in the control group (reduction in lower extremity lymphedema index, 14.7 vs. 6.7; $P=0.03$ ). No unfavorable complications related to PORT were observed in our cohort. 
Conclusion: It would be highly beneficial to combine reduction treatment in the early postoperative period after LVA surgery to maximize treatment outcomes.

Keywords: Lymphedema, complex decongestive therapy, lymphaticovenular anastomosis, reduction treatment, compression, exercise

\section{INTRODUCTION}

The mainstay of lymphedema treatment is complex decongestive therapy (CDT). CDT is a conservative treatment method that includes compression therapy, manual lymph drainage, remedial exercise, and skin care, and it has been proven to be effective in reducing edema ${ }^{[1-4]}$. However, studies have shown that the effectiveness of CDT is dependent on residual lymphatic function ${ }^{[5,6]}$. Furthermore, CDT does not address functional insufficiency in the lymphatic drainage system ${ }^{[7]}$. In contrast, microsurgical treatments, including lymphaticovenular anastomosis (LVA) and vascularized lymph node transfer, increase lymph drainage routes and boost lymphatic drainage, which all play an essential role in improving intrinsic lymphatic function ${ }^{[7]}$.

Studies have indicated the importance of compression therapy after $\mathrm{LVA}^{[7-9]}$; however, no general consensus has been reached regarding when to start and what to combine in the early postoperative period ${ }^{[10]}$. In general, CDT consists of two phases: a reduction phase, followed by a maintenance phase $\mathrm{e}^{[1-3,11]}$. Most patients are likely to undergo LVA surgery during their maintenance phase $\mathrm{e}^{[12,13]}$. According to the literature, after LVA surgery, patients typically resume and continue maintenance treatment, including compression therapy, after 1-4 weeks of postoperative interruption ${ }^{[7,9,10]}$. However, to our knowledge, no study has reported an integration of conservative therapy in the early postoperative period, especially within 1 week after LVA.

In the early postoperative period, we hypothesized that reduction treatment could be performed more efficiently with a boosted lymphatic drainage through the LVA. Based on this hypothesis, we adopted reduction treatment in the early postoperative period after LVA surgery in October 2015. This study aimed to clarify the efficacy of the integration of LVA and perioperative reduction treatment (PORT), in the search for an optimal combination of surgery and conservative therapy for lymphedema, by retrospectively reviewing our experience treating patients with lower extremity lymphedema.

\section{METHODS}

We conducted a retrospective chart review of 134 consecutive patients with lower extremity lymphedema who were treated with LVA, by the same surgeon (FO), at two affiliated hospitals, Saitama Medical Center and Tochigi Cancer Center, between 2014 and 2018. The exclusion criteria were as follows: follow-up period of less than 1 year and patients who did not undergo appropriate compression therapy before and after LVA surgery. Patients with insufficient response to CDT or those whose clinical improvement plateaued following CDT were indicated for LVA surgery. PORT, which included compression therapy and remedial exercise, has been adopted in our clinical practice since October 2015 for use during the early postoperative period immediately following LVA. Prior to this, no additional perioperative intervention had been provided for patients who underwent LVA. In both cases, all patients who underwent LVA surgery wore elastic stockings as baseline maintenance therapy preoperatively for some duration (more than 1 year on average) and resumed wearing the stockings 1 week postoperatively. This study's cohort was divided into two patient groups: patients who underwent PORT following LVA surgery (PORT group) and patients who underwent no additional perioperative intervention after LVA surgery during the early postoperative period 
(control group). The edema reduction effect was compared between the two groups. This study was conducted in accordance with the Declaration of Helsinki and was approved by the Institutional Review Board of Saitama Medical University (\#1483) and Tochigi Cancer Center (\#21-C004).

\section{Surgical procedure}

We performed almost all LVA surgeries under local anesthesia. Three sites for LVA were selected based on preoperative indocyanine green lymphangiography. After making a $2 \mathrm{~cm}$ skin incision, lymphatic vessels and venules were collected nearby, and a side-to-end anastomosis was performed with an 11-0 nylon sutures using a surgical microscope. Upon completion of anastomosis, skin closure was performed using a 5-0 monofilament absorbable subcuticular suture. Prophylactic antibiotics were administered on the day of the surgery. No perioperative anticoagulation therapy was administered to our patients.

\section{Perioperative interventions}

Currently, in our clinical practice, we have adopted reduction treatment, including compression therapy and remedial exercise, in the early postoperative period after LVA since October 2015. Patients underwent compression therapy and exercised under compression from the first postoperative day. Patients wore elastic bandages and/or elastic stockings with a target interface pressure of 20-60 $\mathrm{mmHg}$ measured by our lymphedema therapists using the Pico Press ${ }^{\circledR}$ (Microlab, Padua, Italy). Compression was carefully applied with an even pressure gradient, while avoiding a direct intrusion into the anastomotic sites. The exercise session consisted of treadmill walking, climbing stairs, and an exercise bike for aerobic exercises as well as resistance exercises, including calf raising and squatting. Patients were instructed to exercise with an appropriate intensity, aiming at 3-4 points on the ratings of perceived exertion (modified Borg scale) ${ }^{[14]}$, namely, moderate to somewhat hard. Specifically, for aerobic exercises, we aimed at an intensity that patients could continue for $20 \mathrm{~min}$. For resistance exercises, the load was applied at a level that patients could repeat the exercise 10-20 times without a break. The exercise protocols were adjusted individually to avoid fatigue and muscle pain on the next day. Patients were discharged on the 7th postoperative day and instructed to continue compression therapy and exercises at home.

\section{Data collection}

We collected clinical data, including age, sex, body mass index (BMI), etiology, previous radiotherapy, previous LVA, clinical stage, duration of CDT before LVA surgery, institute of treatment, and measured girth. The clinical stage was based on the classification of the lymph edematous limb set forth by the International Society of Lymphology ${ }^{[1]}$. Girth measurements were performed at five points in the lower extremity: the foot $\left(\mathrm{C}_{\mathrm{F}}\right)$, ankle $\left(\mathrm{C}_{\mathrm{A}}\right)$, calf $\left(\mathrm{C}_{\mathrm{C}}\right)$, knee $\left(\mathrm{C}_{\mathrm{K}}\right)$, and the thigh $(10 \mathrm{~cm}$ above the upper border of the patella bone) $\left(\mathrm{C}_{\mathrm{T}}\right)$. Girth measurement data at the first visit, one month before, and one year after LVA surgery were collected. The extremity volume was estimated using the lower extremity lymphedema index (LEL index $)^{[15]}$ which is given by the formula: LEL index $=\left(\mathrm{C}_{\mathrm{F}}{ }^{2}+\mathrm{C}_{\mathrm{A}}{ }^{2}+\mathrm{C}_{\mathrm{C}}{ }^{2}+\mathrm{C}_{\mathrm{K}}{ }^{2}+\mathrm{C}_{\mathrm{T}}{ }^{2}\right) / \mathrm{BMI}$.

\section{Statistical analysis}

Propensity score analysis was conducted to minimize the effects of selection bias and potential confounders. The propensity score was calculated using a logistic regression model, with the following variables: age, BMI, sex, etiology, clinical stage, LEL index at the first visit, previous radiotherapy, previous LVA, institute of treatment, and duration of preoperative CDT. Multiple logistic regression analysis provided each participant with a propensity score that represented the probability of being treated with PORT following LVA surgery. The nearest neighbor propensity score matching was used to match participants who were treated with PORT and without PORT after LVA, at a 1:1 ratio, with a caliper of 0.2. To assess for differences in demographics and characteristics between the groups before and after matching, we performed the Wilcoxon rank sum test for continuous data and chi-square or Fisher's exact test for 
categorical data. To assess the significance of volume reduction after treatment, LEL indices between 1 month preoperatively and 1 year postoperatively were compared using the paired Wilcoxon rank sum test for both matched groups. Likewise, edema reduction was defined as the difference in LEL indices between 1 month before and 1 year after LVA surgery and was compared between the two groups using the Wilcoxon rank sum test. All tests were two-tailed, and statistical significance was defined as $P<0.05$. All analyses were

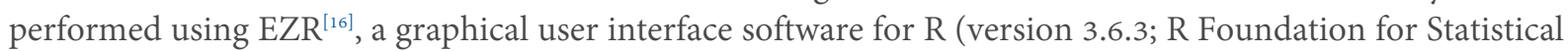
Computing, Vienna, Austria).

\section{RESULTS}

The baseline characteristics and demographics of patients who underwent LVA surgery with and without perioperative reduction therapy are summarized in Table 1 . The current study included 116 patients with lower extremity lymphedema. Six patients (5\%) presented with primary lymphedema and 110 patients (95\%) developed lymphedema secondary to cancer therapy. Among these, 65 patients (56\%) were treated with LVA surgery without perioperative reduction therapy, whereas 51 patients (44\%) were treated with LVA followed by perioperative reduction therapy.

Overall, there was a significant difference between the two unmatched groups at the distribution in clinical stage $(P=0.03)$. That is, all the patient in PORT group were classified as stage II, whereas control group included $6(9.2 \%)$ and 1 (1.5\%) patients of stage I and III, respectively. Other covariates, including age, BMI, sex, etiology, institute of treatment, LEL index at the first visit, previous radiotherapy/LVA, and duration of preoperative conservative therapy, showed a slight difference between the two groups, although this was not statistically significant.

After propensity score matching, patients in each group were evenly matched for age, BMI, sex, etiology, clinical stage, institute of treatment, LEL index at the first visit, previous radiotherapy/LVA, and duration of preoperative conservative therapy. PORT group demonstrated significantly lower LEL indices at 1 year postoperatively than at 1 month preoperatively $(P<0.001)$, whereas the control group did not $(P=0.14)$. Patients who underwent PORT had a significantly higher reduction in LEL index than those in the control group (14.7 vs. 6.7; $P=0.03$ ) [Table 2]. We did not experience any unfavorable complications in our cohort related to PORT, including hemorrhage, wound infection, and wound dehiscence.

\section{Case presentation}

A 63-year-old female patient had bilateral lower extremity lymphedema secondary to cervical cancer and had undergone over 5 years of compression therapy using elastic stockings [Figure 1A]. Preoperative lymphoscintigraphy showed extensive dermal backflow in the right lower leg, right thigh, and left thigh [Figure 2A]. The patient underwent LVA surgery followed by PORT of the right lower extremity during her 7-day hospital stay. After she was discharged from the hospital, compression therapy with elastic stockings was continued. A remarkable improvement in the LEL index, by up to 20.9, was noticeable 1 year postoperatively [Figure $1 \mathrm{~B}$ ]. Lymphoscintigraphy at 6 months after treatment demonstrated a significant reduction in dermal backflow in the right lower extremity [Figure 2B].

\section{DISCUSSION}

This study demonstrated that the treatment efficacy of LVA surgery followed by PORT was significantly higher than that of LVA with no combined therapy in the early postoperative period. 
Table 1. Patient characteristics and demographics before and after propensity score matching

\begin{tabular}{|c|c|c|c|c|c|c|}
\hline & \multicolumn{3}{|c|}{ Before propensity score matching } & \multicolumn{3}{|c|}{ After propensity score matching } \\
\hline & With PORT & Control & P-value & With PORT & Control & P-value \\
\hline Patients, $n$ & 51 & 65 & & 41 & 41 & \\
\hline Age, years (range) & $62.0(32-83)$ & $61.0(26-86)$ & 0.72 & $63.0(32-83)$ & $61.0(30-86)$ & 0.71 \\
\hline $\mathrm{BMI}, \mathrm{kg} / \mathrm{m}^{2}$ (range) & $21.9(18.6-34.1)$ & $23.3(18.1-34.3)$ & 0.58 & $22.0(18.6-34.1)$ & $22.5(18.4-34.3)$ & 0.99 \\
\hline Sex, $n(\%)$ & & & 0.24 & & & \\
\hline Female & $46(90.2)$ & $63(96.9)$ & & $40(97.6)$ & $40(97.6)$ & 1.00 \\
\hline Male & $5(9.8)$ & $2(3.1)$ & & $1(2.4)$ & $1(2.4)$ & \\
\hline Etiology, $n(\%)$ & & & 1.00 & & & 1.00 \\
\hline Primary & $3(5.9)$ & $3(4.6)$ & & $2(4.9)$ & $1(2.4)$ & \\
\hline Secondary & $48(94.1)$ & $62(95.4)$ & & $39(95.1)$ & $40(97.6)$ & \\
\hline Clinical stage, $n(\%)$ & & & $0.03^{*}$ & & & 1.00 \\
\hline । & $0(0.0)$ & $6(9.2)$ & & $0(0.0)$ & $0(0.0)$ & \\
\hline II & $51(100.0)$ & $58(89.2)$ & & $41(100.0)$ & $40(97.6)$ & \\
\hline III & $0(0.0)$ & $1(1.5)$ & & $0(0.0)$ & $1(2.4)$ & \\
\hline $\begin{array}{l}\text { LEL index at the first visit, mean } \\
\text { (range) }\end{array}$ & $\begin{array}{l}279.5(206.0- \\
489.1)\end{array}$ & $\begin{array}{l}267.0(194.8- \\
370.0)\end{array}$ & 0.12 & $\begin{array}{l}277.7(206.0- \\
375.0)\end{array}$ & $\begin{array}{l}259.9(194.8- \\
345.4)\end{array}$ & 0.29 \\
\hline Previous radiotherapy, $n$ (\%) & $14(27.5)$ & $18(27.7)$ & 1.00 & $10(24.4)$ & $12(29.3)$ & 0.80 \\
\hline Previous LVA, $n(\%)$ & $20(39.2)$ & $22(33.8)$ & 0.57 & $16(39.0)$ & $14(34.1)$ & 0.82 \\
\hline Institute of treatment, $n$ (\%) & & & 0.58 & & & 0.83 \\
\hline SMC & $22(43.1)$ & $32(49.2)$ & & $20(48.8)$ & $18(43.9)$ & \\
\hline TCC & $29(56.9)$ & $33(50.8)$ & & $21(51.2)$ & $23(56.1)$ & \\
\hline $\begin{array}{l}\text { Duration of preoperative CDT, month } \\
\text { (range) }\end{array}$ & 19.0 & $18.0(3-360)$ & 0.88 & $14.0(2-161)$ & $18.0(3-360)$ & 0.77 \\
\hline
\end{tabular}

*Statistically significant. PORT: Perioperative reduction treatment; BMI: body mass index; LEL index: lower extremity lymphedema index; LVA: lymphaticovenular anastomosis; SMC: Saitama Medical Center; TCC: Tochigi Cancer Center; CDT: complex decongestive therapy.

Table 2. Outcome comparison between two groups after propensity score matching

\begin{tabular}{llll}
\hline & With PORT $(\boldsymbol{n}=\mathbf{4 1})$ & Control $(\boldsymbol{n}=\mathbf{4 1})$ & $\boldsymbol{P}$-value \\
\hline LEL index at preoperative 1 month, mean (range) & $287.4(199.0-330.1)$ & $261.5(203.8-312.8)$ & $0.048^{*}$ \\
LEL index at postoperative 1 year, mean (range) & $258.4(194.2-333.3)$ & $254.4(181.9-333.9)$ & 0.61 \\
Reduction in LEL index, mean (range) & $14.7(-31.2-70.4)$ & $6.7(-59.8-35.5)$ & $0.03^{*}$ \\
\hline
\end{tabular}

*Statistically significant. PORT: Perioperative reduction treatment; LEL index: lower extremity lymphedema index.

According to Filippetti et al. ${ }^{[17]}$, the benefits of CDT are generated by improving lymph drainage while preventing venous pressure from rising. Studies have shown that a good response to CDT is correlated with a higher remaining lymphatic function ${ }^{[5,6]}$. Therefore, those findings suggest that the basic principle of CDT depends on the remaining lymphatic system. Given that the microsurgical treatment of lymphedema could play a role in improving the intrinsic deteriorated lymphatic function ${ }^{[7,18]}$, it could be hypothesized that CDT, especially reduction treatment, would be more effective, with improved remaining lymphatic function after LVA surgery.

LVA is a microsurgical treatment that redirects excess lymph fluid from the interstitial space to the venous circulation through the bypasses. For better improvement with LVA, lymphatic pressure needs to be higher than venous pressure ${ }^{[1,12,19]}$. In an experimental study, Gloviczki et al. ${ }^{[20]}$ reported that the main problems responsible for occlusion of LVAs were low lymphatic flow, low pressure gradient across the anastomosis, and venous reflux. They mentioned that lymph flow eventually diminished as the anastomosis gradually 

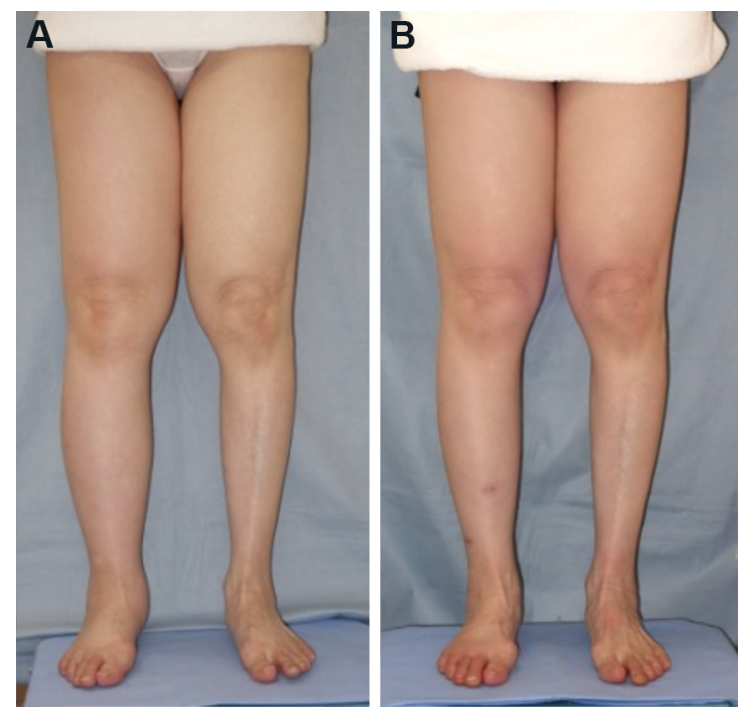

Figure 1. An illustrative case of a 63-year-old woman with bilateral lower extremity lymphedema who underwent perioperative reduction treatment following LVA. (A) 1 month before LVA; (B) 1 year after LVA for the right lower extremity. A remarkable improvement of edema in the right lower extremity was noted. LVA: Lymphaticovenular anastomosis.

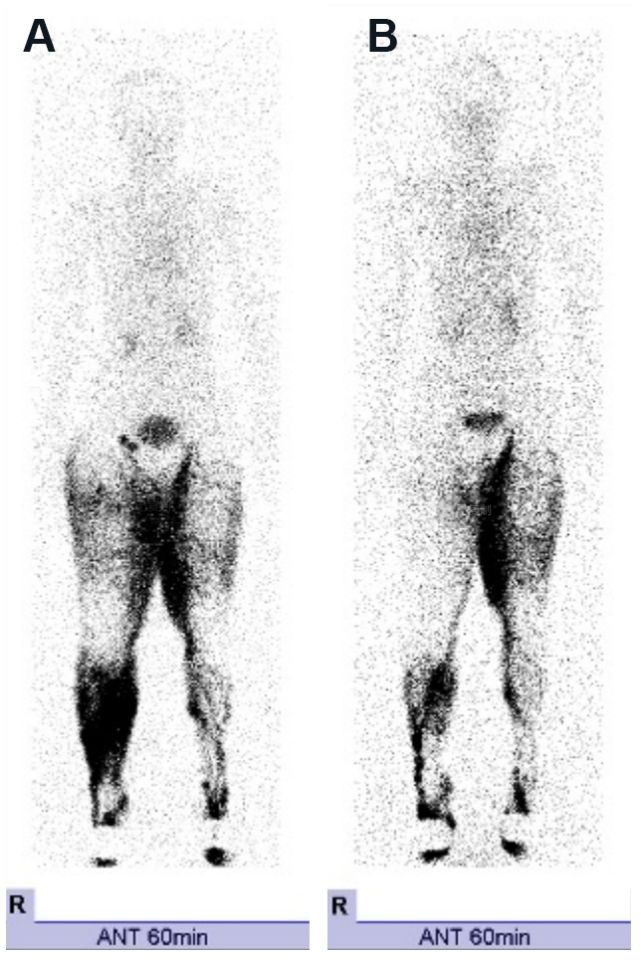

Figure 2. Comparison of lymphoscintigraphy before and after the treatment. (A) 1 month before LVA; (B) 6 months after LVA. Note that the dermal backflow over the right lower leg and the thigh diminished and the lymphatic pathways became distinct after LVA followed by perioperative reduction treatment. LVA: Lymphaticovenular anastomosis.

reduced lymphatic pressure, which usually deteriorates in lymphedema in the first place. According to a systematic review conducted by Tourani et al. ${ }^{[21]}$, the overall long-term patency rate of LVA was discouraging in animal studies due to the gradual decline of the pressure gradient across the anastomosis 
and the eventual thrombosis due to venous reflux. Similarly, several clinical studies have reported that venous reflux diminished the surgical outcome with LVA $^{[22]}$ and caused occlusion of the anastomotic site over time ${ }^{[23]}$.

Tourani et al. ${ }^{[21]}$ mentioned possible technical ideas to overcome the decrease in lymphatic pressure and thrombus formation from venous reflux at the anastomoses; however, they did not include a physiotherapeutic approach. In other words, muscle pumping under compression therapy can amplify lymphatic flow ${ }^{[20,24,25]}$. Chen et al. ${ }^{[26]}$ observed that immediate limb compression following LVA prevented venous backflow by augmenting lymphatic pressure. Likewise, according to Olszewski et al. ${ }^{[25]}$, muscle contraction in the lower leg increased lymphatic pressure and could play an important role as a driving force for lymph flow. They observed that lymphatic pressure could rise as much as $100 \mathrm{mmHg}$ during muscle contraction under compression therapy and that the optimal compression pressure was $40 \mathrm{mmHg}$. It can be hypothesized that this amplified lymphatic flow yielded by muscle pumping under compression therapy could boost bypass flow and overcome venous reflux. Thus, we presumed that LVA followed by reduction treatment in the early postoperative period would be more beneficial than LVA with maintenance treatment resumed after a certain period of interruption.

Generally, LVA is indicated when conservative therapy is proven to be insufficient or plateaued ${ }^{[12,13]}$. Studies have reported that the previous compression therapy, namely maintenance treatment, should be restarted one month postoperatively ${ }^{[7,9,10,27]}$. We believe that this may be a loss of great opportunity for another reduction treatment because the lymphatic-venous pressure gradient and the boosted lymphatic function could be optimal in the early postoperative period with the bypasses. Our findings support the possibility of improving lymphedema more efficiently by combining PORT with LVA. We aimed at the highest interface pressure the patient could tolerate by exerting 20-60 $\mathrm{mmHg}$ using compression therapy, as reported in previous studies ${ }^{[1,25]}$. Additionally, we demonstrated that physical exercise under compression could be combined efficiently and safely as an integral part of PORT. Without a doubt, patients should continue maintenance treatment at home after discharge from the hospital to maintain the reduction in edema.

As previous studies have reported low rates of complications for LVA surgery, such as infection and lymphorrhea ${ }^{[27]}$, our clinical protocol was not accompanied by those complications perioperatively. Undoubtedly, care must be taken to avoid shear stress on the anastomotic site when patients apply compression garments or bandages ${ }^{[28]}$ in the early postoperative period. In addition, remedial exercise is not considered harmful for anastomotic sites unless they are exposed to a traumatic external force. Several review studies have shown that the hospital stay after LVA surgery can be as short as 1 day $^{[29]}$, and patients are assumed to return to their normal daily life. It is presumed that our exercise protocol activity during hospital stay is no more than daily activities. Thus, our study may support the safety of reduction treatment in the early postoperative period.

Our study has some limitations. First, we cannot separately determine the exact efficacy of LVA and that of perioperative reduction treatment because edema reduction after LVA should be an integrated effect of both LVA and CDT. Therefore, further investigation should clarify whether our enhanced treatment efficacy can be attributed to the effect of LVA itself under augmentation with PORT or the effect simply added by CDT. Second, we did not address the long-term patency of LVA. Accordingly, whether perioperative reduction therapy can impact the patency of LVA should be elucidated in future studies. Third, our assessment only focused on the edema reducing effect with the suggested lymphedema treatment. Since lymphedema is often comorbid with acute or chronic inflammation as well as fluid accumulation, further investigation regarding the efficacy of the suggested combined treatment on reducing inflammation is needed. 
In conclusion, our study demonstrated that LVA surgery plus PORT yielded significantly greater edema reduction than LVA alone in the early postoperative period. Based on our findings, it would be highly beneficial to combine reduction treatment during the early postoperative period after LVA surgery to maximize treatment outcomes because the surgical outcomes of LVA alone can be diverse. To our knowledge, our study is the first to describe a combination of LVA and PORT. We believe that this study will contribute to the development of an optimal treatment strategy for patients with lymphedema.

\section{DECLARATIONS}

\section{Authors' contributions}

Conception and design of the study: Onishi F, Tsugu W, Minabe T, Okuda N

Data collection and analysis: Onishi F, Tsugu W, Okuda N

Manuscript writing: Onishi F, Minabe T

\section{Availability of data and materials}

Not applicable.

\section{Financial support and sponsorship}

None.

\section{Conflicts of interest}

All authors declared that there are no conflicts of interest.

\section{Ethical approval and consent to participate}

This study was conducted in accordance with the Declaration of Helsinki and approved by the institutional review board.

\section{Consent for publication}

The authors obtained consent for publication from the patient we present in this article.

\section{Copyright}

(c) The Author(s) 2021.

\section{REFERENCES}

1. Committee. The diagnosis and treatment of peripheral lymphedema: 2016 Consensus Document of the International Society of lymphology. Lymphology 2016;49:170-84. DOI

2. Földi M. Editorial treatment of lymphedema. Lymphology 1994;27:1-5. PubMed

3. Földi E. The treatment of lymphedema. Cancer 1998;83:2833-4. DOI PubMed

4. Kim SJ, Park YD. Effects of complex decongestive physiotherapy on the oedema and the quality of life of lower unilateral lymphoedema following treatment for gynecological cancer. Eur J Cancer Care (Engl) 2008;17:463-8. DOI PubMed

5. Seo KS, Suh M, Hong S, Cheon GJ, Lee SU, Jung GP. The new possibility of lymphoscintigraphy to guide a clinical treatment for lymphedema in patient with breast cancer. Clin Nucl Med 2019;44:179-85. DOI PubMed

6. Yoo J, Choi JY, Hwang JH, et al. Prognostic value of lymphoscintigraphy in patients with gynecological cancer-related lymphedema. $J$ Surg Oncol 2014;109:760-3. DOI PubMed

7. Forte AJ, Khan N, Huayllani MT, et al. Lymphaticovenous anastomosis for lower extremity lymphedema: a systematic review. Indian J Plast Surg 2020;53:17-24. DOI PubMed PMC

8. Cormier JN, Rourke L, Crosby M, Chang D, Armer J. The surgical treatment of lymphedema: a systematic review of the contemporary literature (2004-2010). Ann Surg Oncol 2012;19:642-51. DOI PubMed

9. Winters H, Tielemans HJ, Sprangers PN, Ulrich DJ. Peri-operative care for patients undergoing lymphaticovenular anastomosis: a systematic review. J Plast Reconstr Aesthet Surg 2017;70:178-88. DOI PubMed

10. Winters H, Tielemans HJP, Hameeteman M, et al. The efficacy of lymphaticovenular anastomosis in breast cancer-related lymphedema. Breast Cancer Res Treat 2017;165:321-7. DOI PubMed PMC

11. Kim YB, Hwang JH, Kim TW, Chang HJ, Lee SG. Would complex decongestive therapy reveal long term effect and lymphoscintigraphy predict the outcome of lower-limb lymphedema related to gynecologic cancer treatment? Gynecol Oncol 


\section{2;127:638-42. DOI PubMed}

12. Lee BB, Laredo J, Neville R. Current status of lymphatic reconstructive surgery for chronic lymphedema: it is still an uphill battle! Int $J$ Angiol 2011;20:73-80. DOI PubMed PMC

13. Garza R 3rd, Skoracki R, Hock K, Povoski SP. A comprehensive overview on the surgical management of secondary lymphedema of the upper and lower extremities related to prior oncologic therapies. BMC Cancer 2017;17:468. DOI PubMed PMC

14. Borg GA. Psychophysical bases of perceived exertion. Med Sci Sports Exerc 1982;14:377-81. PubMed

15. Yamamoto T, Matsuda N, Todokoro T, et al. Lower extremity lymphedema index: a simple method for severity evaluation of lower extremity lymphedema. Ann Plast Surg 2011;67:637-40. DOI PubMed

16. Kanda Y. Investigation of the freely available easy-to-use software 'EZR' for medical statistics. Bone Marrow Transplant 2013;48:4528. DOI PubMed PMC

17. Filippetti M, Santoro E, Graziano F, Petric M, Rinaldi G. Modern therapeutic approaches to postmastectomy brachial lymphedema. Microsurgery 1994;15:604-10. DOI PubMed

18. Chen WF, Zhao H, Yamamoto T, Hara H, Ding J. Indocyanine green lymphographic evidence of surgical efficacy following microsurgical and supermicrosurgical lymphedema reconstructions. J Reconstr Microsurg 2016;32:688-98. DOI PubMed

19. Koshima I, Nanba Y, Tsutsui T, Takahashi Y, Itoh S, Fujitsu M. Minimal invasive lymphaticovenular anastomosis under local anesthesia for leg lymphedema: is it effective for stage III and IV? Ann Plast Surg 2004;53:261-6. DOI PubMed

20. Gloviczki P, Hollier LH, Nora FE, Kaye MP. The natural history of microsurgical lymphovenous anastomoses: an experimental study. J Vasc Surg 1986;4:148-56. PubMed

21. Tourani SS, Taylor GI, Ashton MW. Long-term patency of lymphovenous anastomoses: a systematic review. Plast Reconstr Surg 2016;138:492-8. DOI PubMed

22. Visconti G, Salgarello M, Hayashi A. The recipient venule in supermicrosurgical lymphaticovenular anastomosis: flow dynamic classification and correlation with surgical outcomes. J Reconstr Microsurg 2018;34:581-9. DOI PubMed

23. Maegawa J, Yabuki Y, Tomoeda H, Hosono M, Yasumura K. Outcomes of lymphaticovenous side-to-end anastomosis in peripheral lymphedema. J Vasc Surg 2012;55:753-60. DOI PubMed

24. Liao SF, Li SH, Huang HY. The efficacy of complex decongestive physiotherapy (CDP) and predictive factors of response to CDP in lower limb lymphedema (LLL) after pelvic cancer treatment. Gynecol Oncol 2012;125:712-5. DOI PubMed

25. Olszewski WL. Contractility patterns of human leg lymphatics in various stages of obstructive lymphedema. Ann N Y Acad Sci 2008;1131:110-8. DOI PubMed

26. Chen WF, Bowen M, Ding J. Immediate limb compression following supermicrosurgical lymphaticovenular anastomosis - is it helpful or harmful? IMJ 2018;2:1. PubMed

27. Basta MN, Gao LL, Wu LC. Operative treatment of peripheral lymphedema: a systematic meta-analysis of the efficacy and safety of lymphovenous microsurgery and tissue transplantation. Plast Reconstr Surg 2014;133:905-13. DOI PubMed

28. Chang DW, Suami H, Skoracki R. A prospective analysis of 100 consecutive lymphovenous bypass cases for treatment of extremity lymphedema. Plast Reconstr Surg 2013;132:1305-14. DOI PubMed

29. Silva AK, Chang DW. Vascularized lymph node transfer and lymphovenous bypass: Novel treatment strategies for symptomatic lymphedema. J Surg Oncol 2016;113:932-9. DOI PubMed 